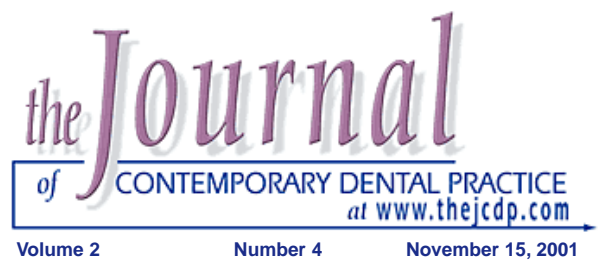

\title{
Selecting Equipment For The Ergonomic Four-Handed Dental Practice
}

\section{Betty Ladley Finkbeiner, CDA, RDA, MS}

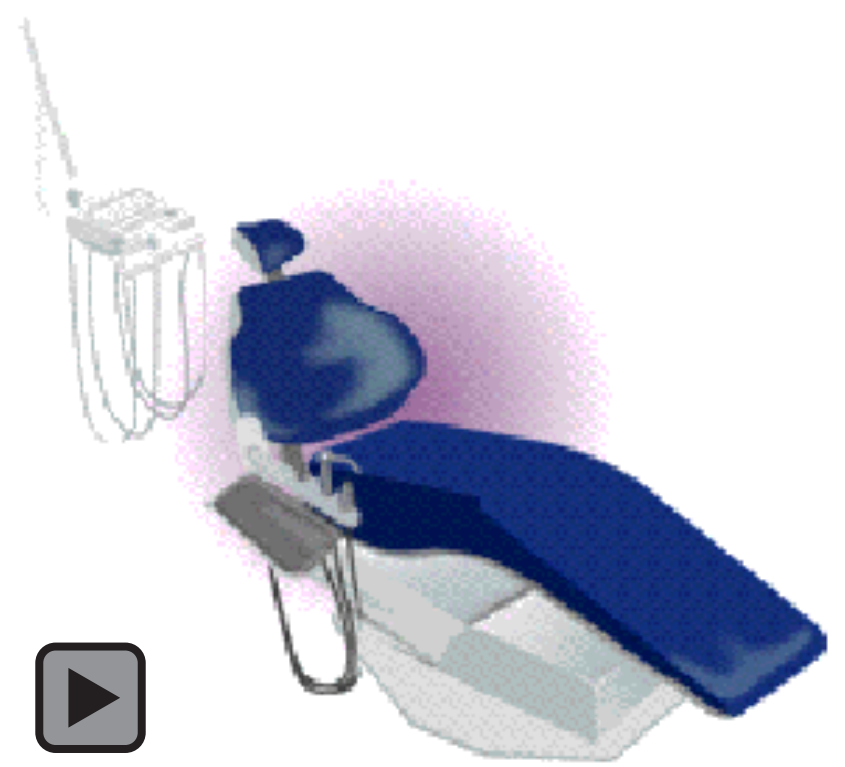

\section{Abstract}

Practicing dental professionals are advised to work with equipment designed to address the need for productivity and stress reduction. Selection of dental equipment for use in an ergonomic, four-handed dentistry practice is integral to the success of the concept. In order for the dental assistant and dentist to work effectively as a team, they have to work in a safe, ergonomically sound working environment. This article examines various designs of dental equipment, the advantages and disadvantages of each, as well as identifies the criteria for equipment selection.

Keywords: Side delivery, rear delivery, split-unit delivery, transthorax delivery, ergonomics

Citation: Finkbeiner BL. Selecting Equipment For The Ergonomic Four-Handed Dental Practice. J Contemp Dent Pract 2001 Nov;(2)4: 044-052.

(C) Seer Publishing 


\section{Introduction}

Four-handed dentistry is ergonomically the most favorable way to provide dental services since it minimizes undesirable movements of the operating team and expedites the progress of most dental procedures.' (Figure 1) To maximize effectiveness, a geographically favorable arrangement of the work environment must exist. The most favorable arrangement requires the selection of equipment be based on the assistant's work area adjacent to the dental chair, since most of the movement of instruments and materials occurs in that area during a treatment procedure.

(Figure 2) Over the years some manufacturers have attempted to meet this criterion and failed. Some of the original research done at the University of Alabama School of Dentistry during the evolution of the concept of fourhanded dentistry during the 1960's is still among the most reliable in meeting these criteria. ${ }^{2}$

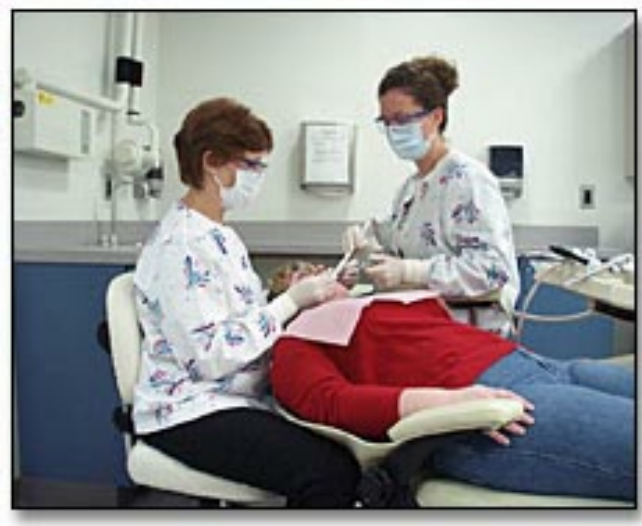

Figure 1.

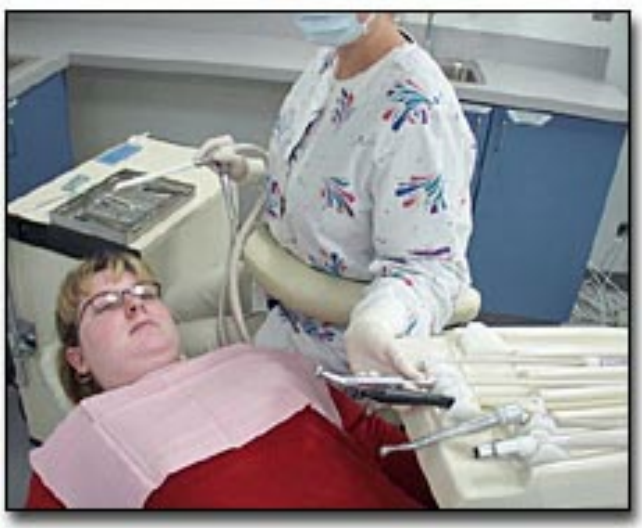

Figure 2.

Ideal fourhanded dentistry simply cannot be performed successfully if the dental team must use excessive movement to reach for instrumentation, become entangled in an array of hoses and power cords, or are unable to comfortably access the operative field. Exhibits at dental conventions display a myriad of equipment styles that can cause a purchaser of new equipment to become confused as to the most favorable equipment to purchase. If inappropriate equipment is purchased, it will likely result in undue stress when used over a period of time.

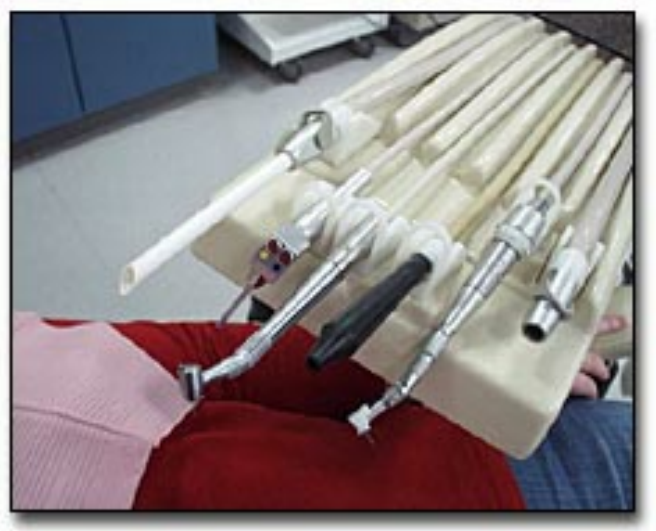

Figure 3.

An often-overlooked factor when choosing a dental unit for ergonomic practice is that the dental assistant should be the primary focus; this will relieve the dentist of unnecessary movement during dental procedures. Accessibility to handpieces and other dynamic instrumentation should be primary to the assistant and secondary to the dentist. ${ }^{2}$ If the dentist must reach to retrieve a handpiece or air-water syringe, time is lost; excessive motion and physical strain can result. ${ }^{3}$ Likewise the position on the unit of the handpieces should be determined by frequency of use. For instance, the high-speed handpiece should be closer to the doctor than the slow speed handpiece, and the high velocity evacuator and air/water syringe should be closer to the assistant than the saliva ejector. (Figure 3) To minimize movements, all instruments should be within a 20 -inch radius of the assistant for efficient instrument transfers to the dentist to occur. ${ }^{1}$

\section{Evaluating Equipment Designs}

The basic dental unit designs available today include the side delivery, rear delivery, split unit/cart, and transthorax. Figure 4 provides schematic examples of the four common unit styles. 


\section{Side Delivery (Figure 4A)}

This unit has been a popular concept for many decades. In fact, several dental schools use this style of unit often supplied with a bracket tray

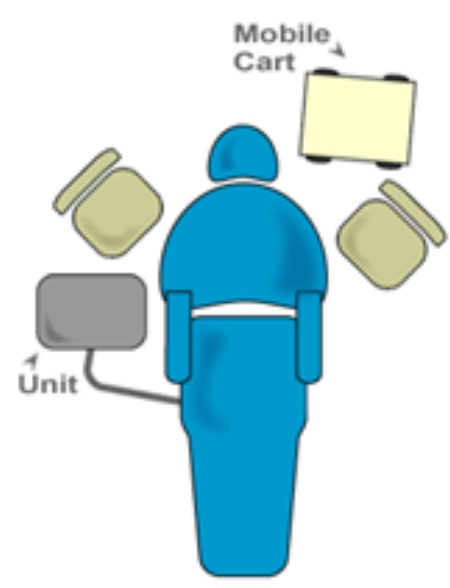

Figure 4A. since dental students often have to work without a dental assistant. Some units of this style even include a cuspidor that interferes with the assistant's position and creates an infection control problem as well as a physical challenge for a patient if they were to actually use it. This style unit requires the dental operator to retrieve and replace the handpieces which, in turn, forces the shift of vision from the treatment site, twists the upper

body to turn to grasp the wanted instrument, and then refocus the eyes back on the operative field. This can result in physical stress and eye fatigue. The side delivery style configuration prohibits the assistant from reaching the instruments to exchange handpieces or change burs that requires more unnecessary movement by the operator, thus, reducing productivity. Often in this arrangement, high velocity evacuation (HVE) is placed on the assistant's side of the chair that, in turn, forces the assistant to be positioned too far away from the patient. At times, HVE hoses are located on the assistant's mobile cart. The concern here is whether the addition of the hoses to this cart diminishes the cart's effectiveness. The hoses may impede the function of a sliding top or encroach on valuable storage space in the cart.

\section{Rear Delivery (Figure 4B)}

The operator is forced to retrieve and replace the handpieces with this style of unit. This requires extensive twisting and turning as well as eyestrain as the operator is forced to turn from the operating field to pick up a handpiece. It is often necessary to transfer the handpiece from the retrieval hand to the operating hand in order to use it. The units are mounted in a fixed position that cannot be moved to accommodate for the changing working positions of the operator or for ease of use for the assistant. HVE hosing and air/water syringes are permanently affixed to an assistant's work area. Since it is in the rear, it requires the assistant to lean forward. When a mobile cart is used with the rear delivery unit concept, it may interfere with access to the sink or to the HVE and air/water syringe. For the assistant, this can provide undue stress and strain and limit access for increased productivity.

Despite the ergonomic disadvantages, rear delivery units remain popular because they are attractive furnishings for the treatment room and hide the dental unit from the patient's view.

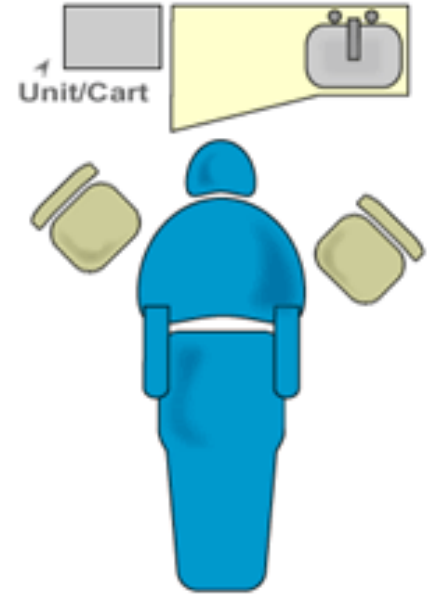

Figure 4B.

\section{Split Unit/Cart (Figure 4C)}

The split unit/cart concept places part of the dental unit on the operator's side and the HVE and air/water syringe on the assistant's mobile cabinet. As in the side delivery unit, it requires the dentist to retrieve the handpieces and makes them inaccessible to the assistant, thus, reducing productivity. The assistant can only use the HVE and the air/water syringe that are attached to the mobile cabinet and is unable to transfer handpieces or change burs for the operator. Often mobile cabinets used in this concept are not designed to contain back-up instruments and adequate storage for dental materials. The split unit design can limit the assistant's working space and requires that back-up instruments be placed in tubs on the fixed cabinetry. This position requires additional motion to retrieve needed instruments and materials that are stored in this manner and opens the door to cross contamination of instruments stored in the tubs.

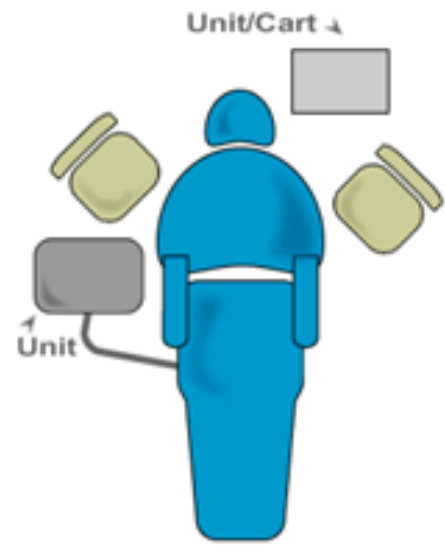

Figure 4C.

\section{Transthorax Unit (Figure 4D)}

The transthorax unit design meets the requirements of favorable time and motion and promotes favorable ergonomics. With the dental unit positioned over the patient's thoracic area, the dental assistant can easily retrieve the handpieces and transfer them to the operator. This eliminates the shift of the operator's vision away from the operat- 
ing site. Repeated shifting of vision from a brightly illuminated operative field at close range to a darker area outside of the oral cavity located at a greater distance forces the eyes of the operator to accommodate for these lighting and distance

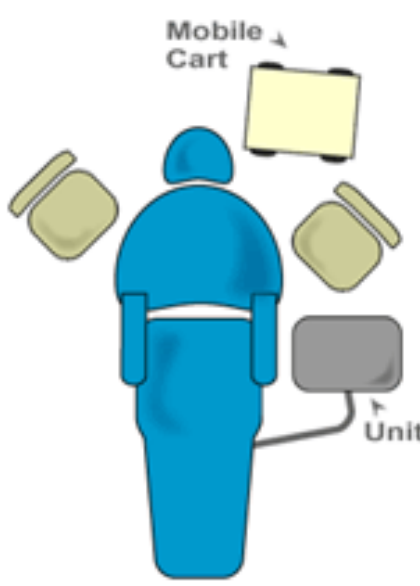

Figure 4D. changes which can lead to eye fatigue and subsequent headache. A mobile cabinet with a moveable top that slides over the seated assistant's knees serves as the primary source of supplies and instruments located well within the comfortable reach of the assistant. There are nit no hoses to interfere with the assistant's position. This type of unit has been designed for the practice of the most effective four-handed dentistry.

\section{Purchasing Equipment}

Purchasing equipment is a long-term investment that must ensure good ergonomics and safe practice for the dental team. As mentioned previously, many dental manufacturers continue to produce antiquated dental units using designs of decades past that include bracket trays and cuspidors. Bracket trays promote the division of instrumentation between the assistant's preset tray and the bracket tray resulting in a scattering of the instruments during a dental procedure. This causes delays in finding and retrieving instruments by both the operator and the assistant. Cuspidors require the removal of isolation materials and raising the patient from the supine operating position in order for them to expectorate. Compared to using the HVE, this is a significant waste of time and inconvenience for the patient.

The dental professional of today must be astute to look for equipment that will maximize productivity and reduce stress to the operating team. It is important to consider patient comfort, but it is unwise to sacrifice operating team comfort that must endure hours of equipment use for an hour of patient comfort during a single appointment. Asking the following questions when selecting dental equipment will be helpful in creating an ergonomically sound work environment through the selection of favorable dental equipment. If the answer is "no" to any of these factors, then it might be wise to search for dental equipment that meets this criteria.

\section{Patient Chair (Figure 5)}

1. Is there a thin narrow back to facilitate access to the patient's oral cavity by the operating team as well as a concave seat and lower lumbar support?

2. Is it free of wide wings that prohibit the assistant and operator from being seated close to the patient?

3. Is there an automatic preset positioner that places the patient in the supine position?

4. Is it free of protrusive devices on the back?

5. Is there provision for neck and head support for the patient?

6. Is it capable of being positioned for right or left handed operators?

7. Is it easily cleanable and free of fabric upholstery?

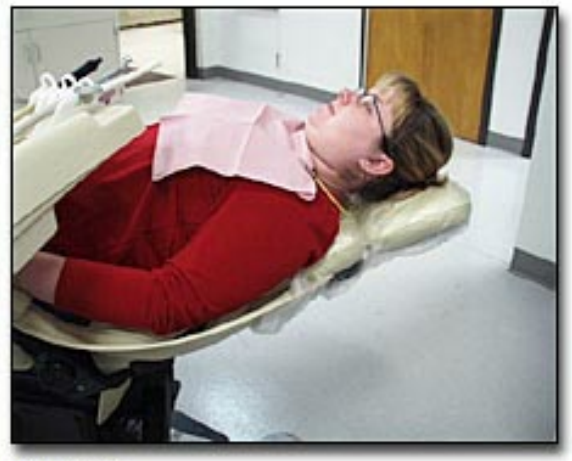

Figure 5.

\section{Dental Unit (Figure 6)}

1. Is it a transthorax style?

2. Does it adjust vertically and have a horizontal tilt for easy access to operator and assistant?

3. Are all of the components, including handpieces, within a 21 inch radius of the assistant? 4. Does it have smooth tubing that is tangle-free, off the floor, and gravity retractable?

5 . Is it able to withstand rigorous daily use?

6. Is it designed to contain multiple handpieces and high technologic devices?

7. Is it free of a bracket table and cuspidor?

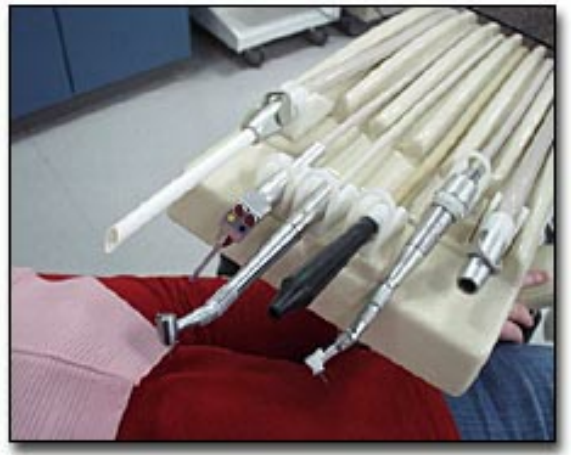

Figure 6. 


\section{Dental Stools (Figure 7)}

1. Do they provide a stable base with four or five casters?

2. Do they have well-padded flat or contoured seats?

3. Are they easily adjustable?

4. Does the assistant's stool provide a back and abdominal support that adjusts vertically and horizontally?

5. Does the operator's stool provide vertical and horizontal adjustment with back support?

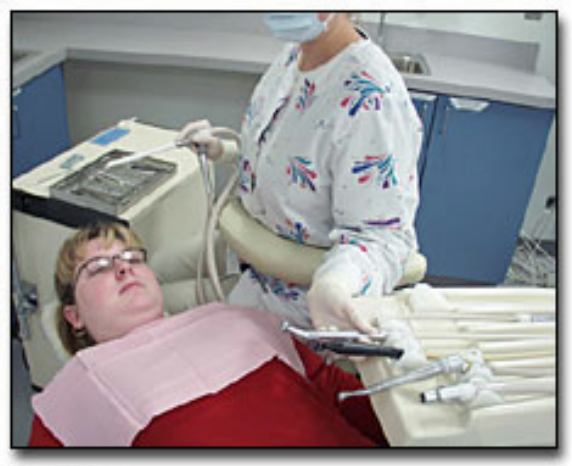

Figure 7.

\section{Mobile Cabinet (Figure 8)}

1. Is it stable and easy to move?

2. Does it provide easy access to instruments and materials?

3 . Is there adequate work surface within 1-2 inches of the elbow?

4. Does it provide a movable work surface that can be positioned over the assistant's lap?

5. Does it provide adequate storage with a deep well at the top?

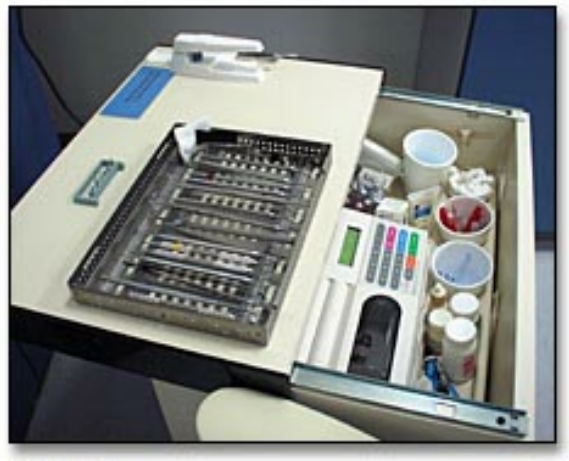

Figure 8.

\section{Fixed Cabinetry}

1. Can it be kept to a minimum?

2. Can it be positioned to provide for maximum floor space?

3. Can it be positioned so the assistant can gain access to back-up materials as needed?
4. Is a section of it located far enough away to avoid infectious aerosols coming in contact with records and radiographs?

\section{Dental Unit Components Including Air/Water Syringe, Handpieces, HVE System}

1. Does each of these components contain lightweight, smooth flexible hosing? (Figure 2)

2. Are they easy to use?

3. Are there fiberoptic options?

4. Can they be easily positioned so that the assistant can readily pass them to the operator without infringing on the patient?

5. Are the attachments to the hoses sterilizable?

6 . Do the handpieces provide a range of speeds and locking mechanisms for hoses to prevent tugback?

7. Does the air/water syringe retain an angled tip that is easily rotated? (Figure 9)

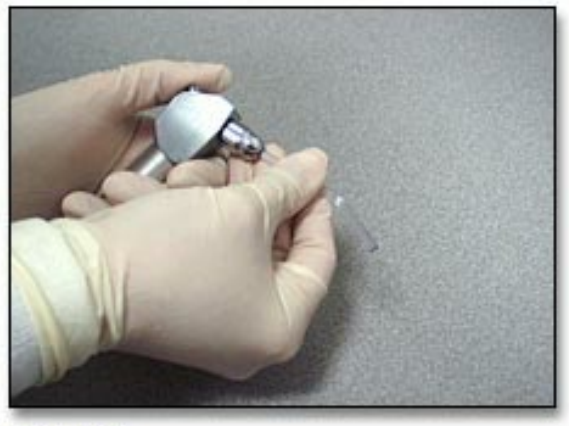

Figure 9.

\section{Conclusion}

Research has shown that ergonomic four-handed dentistry is the most efficient way to create a stress free productive dental practice. It requires the selection of equipment be based on the assistant's domain and good ergonomic practice. Ideal fourhanded dentistry requires that appropriate techniques be followed for seating the patient and operating team, instrument transfer, oral evacuation, and infection control. Furthermore, during all of these procedures attention must be given to conserving motion. It is unwise to continue to work with equipment that does not include ergonomic concepts in its design to promote productivity and reduce stress for the operating team. The assistant and dentist must work as a team, in practice as well as in design of a safe ergonomically sound working environment. 


\section{References}

1. Bramson JB, Smith S, Romagnoli G. Evaluating dental office ergonomic. Risk factors and hazards. J Am Dent Assoc. 1998 Feb;129(2):174-83

2. Murphy, D.C. (1998). Ergonomics and the dental health care worker. Washington, DC: American Public Health Association.

3. Robinson GE, Wuehrmann AH, Sinnett GM, et. al. Four-handed dentistry: the whys and wherefores. J Am Dent Assoc. 1968 Sep;77(3):573-9. No abstract available.

About the Author

\section{Betty Ladley Finkbeiner, CDA, RDA, MS}

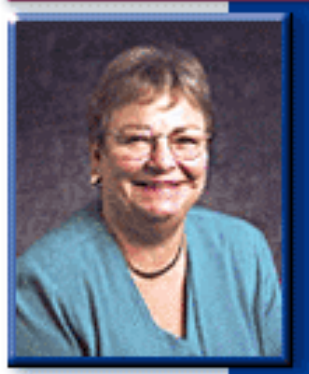

Betty Ladley Finkbeiner is the Chairperson of the Dental Assisting Program at Washtennaw Communtiy College, Ann Arbor, Michigan. She has served as a consultant and staff representative for the American Dental Association's (ADA) Commission on Dental Accreditation and as a consultant to the Dental Assisting National Board. In 1999 she was appointed to the Michigan Board of Dentistry.

Ms Finkbeiner has authored articles in professional journals and co-authored several textbooks including: Practice Management for the Dental Team, Comprehensive Dental Assisting: A Clinical Approach, and Review of Comprehensive Dental Assisting. Most recently she has authored a handbook with Prentice Hall entitled, Four-handed Dentistry: A handbook of Clinical Application and Ergonomic Concepts. She has co-authored videotape productions including Medical Emergencies for the Dental Team, Four-handed Dentistry: An Ergonomic Concept, and Infection Control for the Dental Team.

Correspondence related to this article can be sent to her at the following address:

Whastenaw Community College

4800 E. Huron River Drive

Ann Arbor, MI 48106

e-mail: blf@wccnet.org 